\title{
Feasibility RCT of definitive chemoradiotherapy or chemotherapy and surgery for oesophageal squamous cell cancer
}

\author{
J M Blazeby ${ }^{\star 1,1,2}$, S Strong ${ }^{1,2}$, J L Donovan ${ }^{1}$, C Wilson ${ }^{1}$, W Hollingworth ${ }^{1}$, T Crosby ${ }^{3}$, J Nicklin ${ }^{2}$, S J Falk ${ }^{4}$, \\ C P Barham², A D Hollowood ${ }^{2}$, C G Streets ${ }^{2}$, D Titcomb², R Krysztopik ${ }^{2,5}$, S M Griffin ${ }^{6}$ and S T Brookes ${ }^{1}$ \\ ${ }^{1}$ Centre of Surgical Research, School of Social and Community Medicine, University of Bristol, Bristol BS8 2PS, UK; ${ }^{2}$ Division of \\ Surgery, Head \& Neck, University Hospitals Bristol NHS Foundation Trust, Bristol BS2 8HW, UK; ${ }^{3}$ Velindre NHS Trust, Unit 2 \\ Charnwood Court, Cardiff CF14 2TL, UK; ${ }^{4}$ Bristol Haematology and Oncology Centre, University Hospitals Bristol NHS Foundation \\ Trust, Bristol BS8 2PS, UK; ${ }^{5}$ Royal United Hospital Bath, Bath, BA1 3NG, UK and ${ }^{6}$ Northern Oesophagogastric Unit, Royal Victoria \\ Infirmary, Queen Victoria Road, Newcastle upon Tyne NE1 4LP, UK
}

Background: The optimal treatment for localised oesophageal squamous cell carcinoma (SCC) is uncertain. We assessed the feasibility of an RCT comparing neoadjuvant treatment and surgery with definitive chemoradiotherapy.

Methods: A feasibility RCT in three centres examined incident patients and reasons for ineligibility using multi-disciplinary team meeting records. Eligible patients were offered participation in the RCT with integrated qualitative research involving audiorecorded recruitment appointments and interviews with patients to inform recruitment training for staff.

Results: Of 375 patients with oesophageal SCC, 42 (11.2\%) were eligible. Reasons for eligibility varied between centres, with significantly differing proportions of patients excluded because of total tumour length $(P=0.002)$. Analyses of audio-recordings and patient interviews showed that recruiters had challenges articulating the trial design in simple terms, balancing treatment arms and explaining the need for randomisation. Before analyses of the qualitative data and recruiter training no patients were randomised. Following training in one centre 5 of 16 eligible patients were randomised.

Conclusions: An RCT of surgical vs non-surgical treatment for SCC of the oesophagus is not feasible in the UK alone because of the low number of incident eligible patients. A trial comparing diverse treatment approaches may be possible with investment to support the recruitment process.

The optimal treatment for localised squamous cell carcinoma (SCC) of the oesophagus remains controversial and international practice includes surgery with or without neoadjuvant treatment or an entirely non-surgical approach with definitive chemoradiotherapy (Allum et al, 2009, 2010; Stahl et al, 2010). In the UK 2013 national audit of new patients with localised oesophageal SCC, 50\% received surgical treatment (alone or with neoadjuvant therapy) and $48 \%$ had definitive chemoradiotherapy (National OesophagoGastric Cancer Audit, 2013). Five-year survival rates of $40 \%$ have been achieved with both treatments in single-centre studies but interventions are associated with significant toxicities, morbidity, risk of procedural-related death and detrimental impact on short- and longer-term health-related quality of life (HRQL) (Parameswaran et al, 2008; Hirst et al, 2011; National OesophagoGastric Cancer Audit, 2012). Comparative data are limited as previous early trials struggled to recruit and were closed early, and more recent trials have included different criteria and interventions (Urba et al, 2001; Burmeister et al, 2005; Chiu et al, 2005; Stahl et al, 2005; Bedenne et al, 2007; Teoh et al, 2013). In one trial, all patients received neoadjuvant chemoradiotherapy and those 
responding were then randomised to surgery or chemoradiotherapy. Survival rates were shown to be similar but extrapolation of results is limited owing to the inclusion only of patients responding to chemoradiotherapy (Bedenne et al, 2007). Another was powered to determine equivalence ( $15 \%$ margin) of surgical and nonsurgical treatments and randomised 172 patients to induction chemotherapy followed by chemoradiotherapy or surgery (Stahl et al, 2005). Overall survival was equivalent between treatments, however, the equivalence margin might be considered too broad to exclude a clinically important difference. The third more recent trial randomised 81 patients to surgery alone or definitive chemoradiotherapy, finding no difference in overall survival or HRQL at 2 or 5 years (Chiu et al, 2005; Teoh et al, 2013). The magnitude of difference between the two treatments required to conclude superiority was never reported by the authors as no sample size calculation was provided. In the UK since the OEO2 study, which included both adenocarcinoma and SSC, standard treatment for oesophageal cancer has been chemotherapy and surgery or definitive chemoradiotherapy (National OesophagoGastric Cancer Audit, 2012), and only more recently the Neoscope trial (Gwynne et al, 2011) has opened that aims to compare strategies for preoperative chemoradiotherapy before surgery. There was therefore a lack of high-quality evidence comparing surgical and non-surgical treatments of localised oesophageal SCC meaning that treatment decision-making is currently based on clinician or patient preferences and a trial is needed.

Trials of surgical and non-surgical treatments can be difficult to conduct and recruit into because of the diverse nature of treatments (Cook, 2009). In the UK, these difficulties were overcome in the ProtecT trial that successfully randomised 1500 men between radical surgery, radiotherapy or active monitoring for localised prostate cancer (Donovan et al, 2002). The ProtecT study used detailed screening logs to record patient eligibility and qualitative methods (audio-recordings of consultations, recruiter and patient interviews) to improve recruitment. Subsequent work by Donovan and colleagues has further demonstrated how qualitative research can improve trial conduct, information provision and rates of informed consent (Donovan et al, 2009; Paramasivan et al, 2011; Donovan et al, 2014). Although these methods have been applied to other settings, it is unknown whether it is possible to successfully recruit into a trial of surgical and non-surgical treatment for oesophageal cancer where the treatment-related morbidities are high, long-term outcomes are poor and the impact on HRQL significant. This study, therefore, aimed to determine the feasibility of a main trial of chemotherapy and surgery $v s$ definitive chemoradiotherapy for localised oesophageal SCC.

\section{MATERIALS AND METHODS}

The study included three parts, (i) monitoring multi-disciplinary team (MDT) meetings to determine the number of incident eligible participants with localised oesophageal SCC and final treatment received, (ii) undertaking a pilot RCT with integrated qualitative research to establish whether recruitment was possible and (iii) development of core information sets for surgeons and oncologists to use to explain the two treatments. Parts (i) and (ii) are reported here.

(i) Determination of incident numbers of eligible participants for a main trial. Multi-disciplinary team meeting records from three specialist centres were studied to capture the number of new patients with localised oesophageal SCC potentially eligible for this trial. Two of the MDT meetings considered all referrals and the third only considered patients without metastatic disease suitable for radical treatment. Multi-disciplinary team data were summarised, documenting incident patients, numbers eligible for the trial, reasons for ineligibility and final recommended treatments.

(ii) Pilot RCT with integrated qualitative research. A pilot RCT was set up to assess acceptance of randomisation and to understand clinician and patient treatment preferences. Eligibility criteria included, aged 18 years or older, histologically confirmed oesophageal SCC, sufficient performance status and fitness to undergo surgery or definitive chemoradiotherapy, tumour staged between T2N0M0 and T4N1M0 (where the T4 tumour involved the diaphragmatic crura or mediastinal pleura only), and a total primary tumour and node length of $<10 \mathrm{~cm}$ (Sobin and Wittekind, 2002). Patients were excluded with concomitant or past malignancy within 5 years (except for basal cell carcinoma or SCC of the skin or in situ carcinoma of the cervix), tumour within $2 \mathrm{~cm}$ of cricopharyngeus, tumour including $>2 \mathrm{~cm}$ of gastric wall or previous treatment that compromised the ability to deliver definitive chemoradiotherapy or surgery. Staging was performed with endoscopic ultrasound (EUS), computed tomography of the chest and abdomen, positron emission tomography when available and staging laparoscopy and bronchoscopy in selected patients. All centres measured tumour length on EUS and total length included a measure of the primary tumour and submucosal disease and lymphadenopathy assessed with standard local criteria (Davies et al, 2012).

Integrated qualitative research to optimise trial recruitment. Qualitative research methods were integrated into the pilot RCT to optimise challenges with recruitment. Eligible patients at identified MDT meetings were sent information sheets and invited to clinic where the appointment was audio-recorded. Consultations took place in the usual hospital setting (Donovan et al, 2009). Patients underwent surgical consultations before meeting the oncologists. Once the patient had made a decision about their preferred treatment option or randomisation, they were invited to take part in a semi-structured interview at home so they could provide detailed feedback on the surgery and oncology appointments, their understanding of the trial design, the treatment arms, the acceptability of randomisation, preferences and their treatment decision.

Audio-recordings and interview data were transcribed verbatim and analysed using techniques of constant comparison to understand how the study was being presented and discussed with patients (Glaser, 1998). Targeted conversation analysis was used to identify problematic aspects of the clinical interaction (Donovan, et al, 2002). The study planned to discuss findings within each centre with individual recruiters and in group training. Group training was based on analysis of the audio recordings (JLD, SS and CW). Standard thematic methods of qualitative data analysis were used to identify key findings that were then presented to surgeons, oncologists and nurses in the recruiting team with illustrative quotes to promote discussion of 'good' and 'not so good' information provision. Full details of this process are described elsewhere and have been integrated into several RCTs (Donovan et al, 2014).

Trial treatments. Induction chemotherapy was given as 21 days of either cisplatin $80 \mathrm{mg} \mathrm{m}^{-2}$ by intravenous infusion on days 1 and 5 followed by fluorouracil $1 \mathrm{~g} \mathrm{~m}^{-2}$ per day as a continuous intravenous infusion for four days or cisplatin $80 \mathrm{mg} \mathrm{m}^{-2}$ by intravenous infusion on day 1 and capecitabine $625 \mathrm{mg} \mathrm{m}^{-2}$ orally twice daily continuously. In the surgical arm, two- or three-phase oesophagectomies were performed as an open, laparoscopically assisted or with a totally minimally invasive approach with a twofield lymphadenectomy. These are described in detail elsewhere (Blazeby et al, 2011). 
Induction chemotherapy before chemoradiotherapy consisted of two cycles of 21 days with either cisplatin $80 \mathrm{mg} \mathrm{m}^{-2}$ by intravenous infusion on day 1 and 5-fluorouracil $1 \mathrm{~g} \mathrm{~m}^{-2}$ per day by continuous intravenous infusion for four days starting on day 1 (i.e., total cycle dose $=4 \mathrm{~g} \mathrm{~m}^{-2}$ ) or cisplatin $80 \mathrm{mg} \mathrm{m}^{-2}$ to be by intravenous infusion on day 1 and capecitabine $625 \mathrm{mg} \mathrm{m}^{-2}$ orally twice daily within $30 \mathrm{~min}$ of food, starting in the evening of day 1 and finishing on the morning of day 43. The chemotherapy component of the definitive chemoradiotherapy was identical to the induction chemotherapy regimen above continued for a further two cycles (i.e., from day 43 to day 84). Radiotherapy was started on day 43 and delivered in a single phase: 50 Gy in 25 fractions.

Outcome assessment and sample size. When eligible patients consented to randomisation, treatment allocation was determined by an automated randomisation web-based system. The proportion of eligible patients consenting to randomisation was examined and treatments recommended for eligible but non-randomised patients noted. The purpose of this feasibility study was to establish potential rates of eligible patients and of those, the percentage consenting to randomisation. Hence, eligibility and randomisation rates were the primary outcomes for this study and were calculated separately for each centre and overall. Differences between centres were examined using Chi-square tests. The pilot RCT assessed the feasibility of data collection including toxicity, surgical morbidity and HRQL (EORTC QLQ-C30 and QLQ-OG25) data (Aaronson et al, 1993; Lagergren et al, 2007). It was not the intention of this study to examine the comparative effectiveness of the two treatments, hence no formal sample size calculation was performed. North Somerset and South Bristol Research Ethics Committee (09/H0106/69) approved this study.

\section{RESULTS}

Centres recruited for different lengths of time between April 2010 and March 2013 (for a total of 82 centre months) during which 375 patients with oesophageal SCC were discussed in 331 MDT meetings. Only 42 (11\%) were considered eligible (Figure 1); this equates to just one eligible patient identified for every two centre

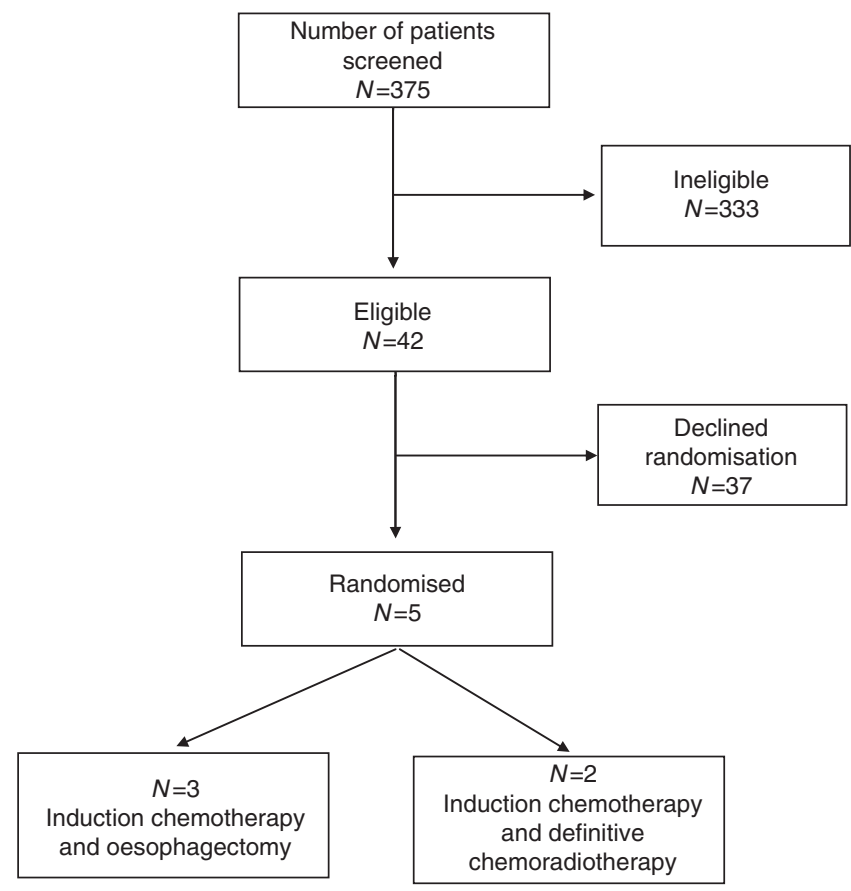

Figure 1. Flow diagram of recruitment into the pilot RCT. months of recruitment. Eligible patients were predominantly women 27(64.3\%) (Table 1).

(i) Determination of incident numbers of eligible trial patients for a main trial. The percentage of eligible patients ranged from $6.0 \%$ to $14.7 \%$ across centres. Further investigation into reasons for ineligibility showed that as expected about a third of patients were not eligible because of metastatic disease and another quarter were ineligible because of frailty (this was lower as expected in the centre that only discussed patients selected for radical treatment). There were unexpected differences between centres, however, in terms of the percentage of patients categorised ineligible because the total tumour and node length exceeding $10 \mathrm{~cm}$, with $27 / 147$ (18.4\%), 4/47 (8.5\%) and 8/139 (5.8\%) of patients in centres 1,2 and 3 , respectively, being classified as ineligible for that reasons, $P=0.002$ (Table 2 ).

(ii) Pilot RCT with integrated qualitative research. In total, 26 paired audio-recordings ( 7 from centre 1, 0 from centre 2 and 19 from centre 3) of surgery and oncology appointments were analysed alongside 14 in-depth patient interviews. Centres 1 and 2 did not randomise any patients and centre 3 randomised five patients. Feedback meetings to improve consultations were undertaken in centres 1 and 3 . In centre 1 following feedback, no eligible patients were identified before the end of the study, preventing analyses of the impact of feedback and training. In centre 3, before feedback no one had consented to randomisation (of 8 eligible patients) but following feedback, 5 of the next 16 eligible patients consented to randomisation (Table 1). This indicates the positive impact on recruitment of training and feedback from the qualitative study.

Analyses of the final treatment received for eligible but nonrandomised patients in centre 1 showed a preference for surgery (Table 2), whereas the proportions of eligible but non-randomised patients in centres 2 and 3 were similar for surgery and definitive chemoradiotherapy.

The qualitative research identified three issues hindering recruitment. Recruiters had difficulties in (a) articulating the trial design in simple terms; (b) balancing treatment arms, in particular, describing the effect of radiotherapy on the tumour; (c) explaining the selection of treatment by randomisation. Difficulties explaining the trial design identified in the early recruitment appointments occurred because recruiters presented patients with the two different treatment options (chemotherapy and surgery or chemo-radiotherapy) without mentioning the trial. As a result, patients thought they had to make a choice between these two treatment options. Other patients reported not understanding what taking part in the trial actually meant. In many appointments, the trial was presented as a third option, only suitable for those who could not make a decision about their preferred treatment (Figure 2 provides illustrative quotes). This limited the number of patients who considered taking part in the study.

Many patients who declined to take part in the study expressed a strong preference, either for or against surgery. Patients intuitively understood that surgery 'removes' or 'cuts out' the tumour, but were uncertain about the action of radiotherapy on the cancer. Recruitment appointments were reviewed to examine how consultants talked about surgery and radiotherapy. In early recruitment appointments, several surgeons unintentionally expressed a preference for surgery. Following training, surgeons were able to use balancing statements to convey the strengths and limitations of both treatment options and to explain the effect of radiotherapy on the tumour (Figure 3 provides illustrative quotes).

Interview data showed that the majority of patients did not like the idea of a computer allocating their treatment and they did not understand the need for randomisation. Early recordings showed consultants were reluctant to explain the rationale for randomisation. Training was provided to help consultants explain 


\section{Table 1. Eligible patient details and final treatment received}

\begin{tabular}{|c|c|c|c|c|}
\hline & Centre $1, N=15$ & Centre $2, N=3$ & Centre $3, N=24$ & Total, $\mathbf{N}=\mathbf{4 2}$ \\
\hline Mean age, years (range) & $67(48-76)$ & $61(55-69)$ & $68(41-77)$ & $65(41-78)$ \\
\hline Female, $n(\%)$ & $10(66.7)$ & $3(100)$ & $14(58.3)$ & $27(64.3)$ \\
\hline \multicolumn{5}{|l|}{ Tumour stage } \\
\hline I & 0 & 0 & 2 & 2 \\
\hline Ila & 1 & 2 & 7 & 10 \\
\hline $\mathrm{llb}$ & 2 & 0 & 14 & 16 \\
\hline III & 13 & 1 & 1 & 15 \\
\hline Randomised into pilot RCT, $n(\%)$ & 0 & 0 & $5(20.8)$ & $5(11.9)$ \\
\hline \multicolumn{5}{|c|}{ Treatment received by eligible but non-randomised patients, $n(\%)$} \\
\hline Chemotherapy \& surgery & $8(53.3)$ & $1(25.0)$ & $7(43.7)$ & $16(43.2)$ \\
\hline Surgery alone & $1(6.7)$ & $0(0.0)$ & $0(0.0)$ & $1(2.7)$ \\
\hline Definitive chemoradiotherapy & $6(40.0)$ & $2(50.0)$ & $9(47.4)$ & $17(45.9)$ \\
\hline Stent & $0(0.0)$ & $0(0.0)$ & $2(10.5)$ & $2(5.4)$ \\
\hline Unknown & $0(0.0)$ & $0(0.0)$ & $1(5.2)$ & $1(2.7)$ \\
\hline
\end{tabular}

Table 2. Numbers of incident patients with oesophageal SCC eligible for the trials, reasons for ineligibility and final treatment received by centres

\begin{tabular}{|c|c|c|c|c|}
\hline & Centre 1, N (\%) & Centre 2, N (\%) & Centre $3, N(\%)$ & Total, $N(\%)$ \\
\hline Incident oesophageal SCC discussed at MDT & 162 & 50 & 163 & 375 \\
\hline Eligible for the pilot RCT & $15(9.3)$ & $3(6.0)$ & $24(14.7)$ & $42(11.2)$ \\
\hline \multicolumn{5}{|l|}{ Reasons for ineligibility ( $n(\%)$ of those not eligible) } \\
\hline $\begin{array}{l}\text { Metastatic disease } \\
\text { Unfit for radical treatment } \\
\text { Total tumour length }>10 \mathrm{~cm} \\
\text { T4 disease (aorta/pericardium) } \\
\text { Tumour }<2 \mathrm{~cm} \text { cricopharyngeus } \\
\text { Previous malignancy } \\
\text { Recurrent disease } \\
\text { Other }\end{array}$ & $\begin{array}{c}44(29.9) \\
43(29.3) \\
27(18.4) \\
14(9.5) \\
4(2.7) \\
7(4.8) \\
3(2.0) \\
5(3.4)\end{array}$ & $\begin{array}{c}15(31.9) \\
9(19.1)^{\mathrm{a}} \\
4(8.5) \\
8(17.0) \\
1(2.1) \\
3(6.4) \\
3(6.4) \\
4(8.5)\end{array}$ & $\begin{array}{c}44(31.7) \\
41(29.5) \\
8(5.8) \\
16(11.5) \\
6(4.3) \\
3(2.2) \\
5(4.0) \\
16(11.5)\end{array}$ & $\begin{array}{c}103(30.9) \\
93(27.9) \\
39(11.7) \\
38(11.4) \\
11(3.3) \\
13(3.9) \\
11(3.3) \\
25(7.5)\end{array}$ \\
\hline
\end{tabular}

randomisation, significantly improving patient understanding and acceptance of the study (Figure 3 provides illustrative quotes).

All participating patients completed HRQL outcome measures at the required time points. Other outcomes were reviewed at clinic appointments to 6 months after randomisation.

\section{DISCUSSION}

This paper describes feasibility work to establish whether a main trial comparing a surgical and non-surgical approach for oesophageal SCC is possible. It integrated specific methodological techniques to optimise recruitment because of the well-recognised challenges enroling patients in trials comparing diverse treatment options and to help surgeons with this unfamiliar process. The study scrutinised MDT records for eligible patients, carefully documenting reasons for ineligibility and it used qualitative research methods to understand how the trial was discussed by surgeons and oncologists and to allow feedback and training. It was found that $11 \%$ of all patients with oesophageal SCC were eligible for the trial, which in this study equated to just one patient every two months that a centre was open for recruitment. Notable differences were seen between the centres in terms of the reasons for ineligibility. Initially, no eligible patients consented to randomisation. In one centre where the qualitative research was effectively applied and feedback meetings undertaken some 5 of the 16 patients were randomised providing an indication that recruitment was possible with appropriate support. Despite the successful randomisation in one centre this study has shown that a full trial comparing a surgical and non-surgical approach for oesophageal SCC is not feasible in the UK alone because of insufficient eligible patients. It does, however, show that recruitment difficulties are often multi-factorial and a range of methods is needed to identify and address them. It also shows how investment with qualitative research in a trial can support surgeons and oncologists to recruit and this method has promising implications for other settings.

The methods used to examine incident patients via MDT meetings were found to be practical and useful in providing accurate information to inform whether a main trial is possible. Others have explored the role of MDT records to optimise trial recruitment and demonstrated that these provide an excellent resource to identify eligible patients into oncology trials (McNair et al, 2008). It is recommended, therefore, that this approach is 
Example quotes before training:

Surgeon 1: "If you can't decide, go into the trial."

Surgeon 2: "If you say that you were happy to go into the trial at this point, you say, 'I don't know which way to go -l'll go into a trial', your name is drawn out of a hat as to which of those two options you get -as you could have either of the two options anyway."

Patient ( 79 yr old male, not consented, surgery preference): "I'm not quite with that word trial at the moment 'cause if you're gonna try something then you do something differently, so what's different? (ctd...) This word trial to me seems to be a completely wrong word. It's not a trial, it's actually what you're gonna get. It's the actual treatment, isn't it?"

Training points: Consultants were asked to replace the word trial with 'study' and to use the study to frame discussion of the treatment options.

Example quote post training:

Surgeon 3: “.... there are different types of treatment for the cancer you have.

You can have chemotherapy and radiotherapy. You can have chemotherapy and

surgery. And the difficulty we have as experts is that we don't know which one of

those treatments is best, so we've started this study...now what I wanted to talk

to you about today is whether or not you would consider going into the

study...you don't have to, it's completely voluntary."

Figure 2. Example quotes to show the difficulties that surgeons had in explaining the study design and the improvements made after training. used more widely. Analysing reasons for ineligibility for the trial also provided useful data. It showed how centres differently used local staging investigations to assess tumour lengths and trial eligibility. For example, centre 1 found a larger proportion of ineligible patients due to disease length than the other centres because of the interpretation of the EUS data. In a main trial more detailed guidance for assessing the tumour length and trial eligibility is recommended.

Other trials have integrated qualitative research methods to optimise recruitment by audio-recording consultations, analysing data and providing training and feedback to surgeons (Donovan et al, 2002; Lewin et al, 2009; Paramasivan et al, 2011; Donovan et al, 2014). To achieve this, however, it is necessary to ensure that audio-recordings are undertaken and returned from the participating centres. Difficulties in obtaining audio-recordings from clinical centres identified in this study have been experienced in another trial of a surgical and non-surgical treatment for bladder cancer (Paramasivan et al, 2011). The reasons for lack of audio-recording consultations and transfer of data may be related to logistical issues. There is evidence that there is a general reluctance among recruiters to be audio-

Example quotes about unbalanced information provision:

Surgeon 4: "I don't think these things are set in stone but if we were going to make a list of treatments starting with which one is most likely to cure my cancer then it's probably gonna be chemotherapy followed by surgery. If we do anything less it won't cure your cancer... and the cancer will eventually kill you."

Surgeon 5: "There is evidence that having chemotherapy and radiotherapy without an operation may also be able to treat the tumour."

Oncologist 1: "There are two treatments for your complaint...either an operationwhere they cut it away or radiotherapy, and chemotherapy where we treat people with $x$-ray treatment and drugs."

Training points:

Consultants were asked to use balancing statements to compare the advantages and disadvantages of both treatments and

to describe the action of the radiotherapy, as being able to "destroy" or "kill" the cancer cells.

Example quotes post training:

Surgeon 4: "We have a treatment that could involve chemotherapy and then an operation to cut it out and we have a treatment that involved chemotherapy and radiation, and our previous experience of patients is that we know that both of those work and as best we can tell, both of them seem to work equally effectively in curing your cancer."

Surgeon 3: "We give radiotherapy to kill off the cancer cells that maybe there."

Example quotes of difficulties explaining randomisation:

Surgeon 1: "If you're not sure of one, we recommend that the decision about which treatment you have is made by a process called randomisation so rather than me, you or $\operatorname{Dr}$ (Surname) deciding, a computer decides and then you're allocated to one of those two, and then, you have got a good chance of having the best treatment because you don't know what's the best treatment. You've got a fifty/fifty chance of having the best treatment. In other words, you don't decide - the computer decides for you."

Example quotes showing patient difficulties to understand/accept randomisation

Patient (77 yr old male, non-randomised, chemo-radiotherapy preference): "[Consultant X] said they put all the information they've got - how old I am and what - where it is into a computer, and then the computer shoots out a result which is the best way to go. No, I don't think that I'd want to be at the mercy of a computer. I wouldn't accept any of that... you see were not computer oriented. We haven't got one in our house."

Patient (71 yr old female, non-randomised, surgery preference): "Why can't they follow people up, whatever option they choose? I don't want a computer to take over my life, make decisions. When I made the choice - right or wrong, l've made it and it's me that's made it."

Training points: Consultants were asked to avoid using the word computer and explain the rationale for randomisation

Example quotes post training:

Oncologist 1: "The study decides whether you have the radiotherapy or the surgery and the reason we have to do that is that the problem is that say we see 10 patients in a row and we say well quite like - the surgeon says well I quite like the look at that one for an operation, they're the right, size, shape and age then that puts in what's called bias into the study. So it has to be done on what's called a random allocation where if you like the study decides rather than we do because otherwise we put bias in it. So if you look at previous studies for example where they've done that the patients operated on tend to be young and fit and the people who have the radiotherapy tend to be older andless fit."

Surgeon 4: "the way of approaching any study would be to allocate the treatment to you, to randomise you to one or the other options and that is done as part of the study itself.... we obviously monitor you as you go through everything, looking at you, the effects of the treatment and see how you are, hopefully at the end of all of this, with enough people we will answer the question as to which one would appear the better option.. So that's the basis of the study itself. If people, if some people don't want to enter into the study then obviously we've got to still think about you and how we would treat you and we're still then left with the same two options."

Figure 3. Example quotes to show the difficulties consultants had in balancing the treatment groups and explaining randomisation, and how the improvements were demonstrated after training. 
recorded (Donovan et al, 2014) and this was also found in this trial. In centres where this was not challenged, recording was not undertaken (centre 2), but when the recording was undertaken by the PI and strongly encouraged, it became a routine (centre 3). These experiences emphasise the need for better training of clinicians before participation in trials and strong support and trial leadership during the study itself.

Recent evidence suggests that there are some generic lessons that can be learned from previous qualitative research integrated with trials, such as the need to support recruiters in explaining aspects of trial design including randomisation and equipoise, and the need to explore the basis of apparent preferences; but also that there are likely to be some issues specific to the particular trial that are likely to require the dedicated qualitative research (Donovan et al, 2014). As more of this research is conducted, it is also possible that clinicians exposed to the training may acquire recruitment skills that will be transferable to other trials. Other methods to improve trial recruitment could involve holding joint clinics with surgeons and oncologists together or changing the order in which patients meet each specialist (Paramasivan et al, 2011). These approaches may be interesting to explore in future work. There has also been an initiative promoted by the Royal College of Surgeons of England for surgical trainees to be involved and recruit into trials in surgery, which has had promising early results (Bhangu et al, 2013).

Few trials have directly compared surgical vs non-surgical treatment for oesophageal cancer, although two RCTs randomised patients following neoadjuvant chemoradiotherapy to consolidation chemoradiotherapy or surgery (Stahl et al, 2005; Bedenne et al, 2007). The original trials of radical surgical and non-surgical approaches initiated some time ago were stopped because of poor recruitment (Urba et al, 2001; Burmeister et al, 2005) and the more recent Chinese trial did not report a sample size calculation recruiting only 80 patients (Chiu et al, 2005; Teoh et al, 2013). In the trials where all patients started chemoradiotherapy recruitment were not reported to be difficult maybe because both trial arms received similar therapies to start with, which may make participation more acceptable to patients and clinicians. The increasing use of chemoradiotherapy before surgery has been shown to convey a survival benefit over surgery alone (van Hagen et al, 2012). Trials show that a pathological complete response rate of up to $25 \%$ may be obtained following neoadjuvant chemoradiotherapy, further raising the question of whether surgery is always needed following chemoradiotherapy for optimal survival benefit. In the UK, the recent SCOPE I trial showed that a 2-year survival rate of $56 \%$ could be achieved following definitive chemoradiotherapy (Crosby et al, 2013). In all these trials, patients with both oesophageal SCC and adenocarcinoma were included and no survival differences were observed by histological cell type. It may therefore be possible that a potential future trial could be designed to compare neoadjuvant chemoradiotherapy and surgery with definitive chemoradiotherapy in patients with both SCC and adenocarcinoma meaning that large numbers of patients would be eligible. Work being initiated within the Neoscope study will help standardise the delivery of preoperative chemoradiotherapy in the UK with an emphasis on safety of the tri-modality therapy (Gwynne et al, 2011). This will contribute to the future development of a surgical $v s$ non-surgical trial in oesophageal cancer.

This feasibility work has shown how MDT records can usefully inform trial recruitment and that the application of qualitative research to optimise trial recruitment is beneficial. Despite the difficulties encountered in this feasibility study, in the centre where audio-recordings were obtained and there was feedback with further patients consulted, some 5 patients were recruited from 16 eligible participants. A full trial comparing surgery with definitive chemoradiotherapy for oesophageal SCC alone, however, is not possible in the UK alone and would require an international effort. The integration of qualitative research methods with accompanying feedback can improve recruitment into trials with very different treatment interventions and should be routinely incorporated.

\section{ACKNOWLEDGEMENTS}

This article summarises independent research funded by the National Institute for Health Research (NIHR) under its Research for Patient Benefit (RfPB) Program (Grant reference PB-PG-080714131).

\section{AUTHORS CONTRIBUTIONS}

JMB, STB, JLD, WH, TC and SMG designed the study with contributions to its adaptation during delivery of the trial from SS, CW, SJF, CPB, ADH, CGS, DT, RK and JN. Participation in recruitment, delivery of interventions and audio-recordings were undertaken by CPB, ADH, RK, CGS, DT, JMB, TC, JN and SMG. SS, CW and JLD analysed and interpreted the qualitative data. The first draft of the paper was written by JMB. All authors contributed to the final version. JMB is the guarantor of the study.

\section{DISCLAIMER}

The views expressed in this publication are those of the author(s) and not necessarily those of the NHS, the NIHR or the Department of Health.

\section{REFERENCES}

Aaronson NK, Ahmedzai S, Bergman B, Bullinger M, Cull A, Duez NJ, Filiberti A, Flechtner H, Stewart B, Fleishman SB, de Haes JCJM, Kaasa S, Klee M, Osoba D, Razavi D, Rofe PB, Schraub S, Sneeuw K, Sullivan M, Takeda F; for the European Organization for Research and Treatment of Cancer (1993) The European Organization for Research and Treatment of Cancer QLQ-C30: a quality-of-life instrument for use in international clinical trials in oncology. J Natl Cancer Inst 85: 365-376.

Allum WH, Stenning S, Bancewicz J, Clark PI, Langley RE (2009) Long term results of a randomized trial of surgery with or without preoperative. J Clin Oncol 27: 5062-5067.

Allum AW, Blazeby JM, Griffin SM, Cunningham D, Jankowski JA, Wong R. Association of Upper Gastrointestinal Surgeons of Great Britain and Ireland, the British Society of Gastroenterology and the British Association of Surgical Oncology (2010) Guidelines for the management of oesophageal and gastric cancer. Gut 60: 1449-1472.

Bedenne L, Michel P, Bouché O, Milan C, Mariette C, Conroy T, Pezet D, Roullet B, Seitz JF, Herr JP, Paillot B, Arveux P, Bonnetain F, Binquet C (2007) Chemoradiation followed by surgery compared with chemoradiation alone in squamous cancer of the esophagus: FFCD 9102. J Clin Oncol 1 25(10): 1160-1168.

Blazeby JM, Blencowe NS, Titcomb DR, Metcalfe C, Hollowood AD, Barham CP (2011) Demonstration of the IDEAL recommendations for evaluating and reporting surgical innovation in minimally invasive oesophagectomy. Br J Surg 98: 544-551.

Bhangu A, Kolias AG, Pinkney T, Hall NJ, Fitzgerald JE (2013) Surgical research collaboratives in the UK. Lancet 382(9898): 1091-1092.

Burmeister BH, Smithers BM, Gebski V, Fitzgerald L, Simes RJ, Devitt P, Ackland S, Gotley DC, Joseph D, Millar J, North J, Walpole ET, Danham JW; Trans-Tasman Radiation Oncology Group; Australasian Gastro-Intestinal Trials Group (2005) Surgery alone versus chemoradiotherapy followed by surgery for respectable cancer of the oesophagus: A randomized controlled phase III trial. Lancet Oncol 6(9): 659-668.

Chiu PW, Chan A, Leung SF, Leong HT, Kwong KH, Li MK, Au-Yeung AC, Chung SC, Ng EK (2005) Multicenter prospective randomized trial 
comparing standard esophagectomy with chemoradiotherapy for treatment of squamous esophageal cancer: early results from the Chinese University Research Group for Esophageal Cancer (CURE). J Gastrointest Surg 9: 794-802.

Cook JA (2009) The challenges faced in the design, conduct and analysis of surgical randomised controlled trials. Trials 10: 9 .

Crosby T, Hurt CN, Falk S, Gollins S, Mukherjee S, Staffurth J, Ray R, Bashir N, Bridgewater JA, Geh JI, Cunningham D, Blazeby J, Roy R, Maughan T, Griffiths G (2013) Chemoradiotherapy with or without cetuximab in patients with oesophageal cancer (SCOPE1): a multicentre, phase 2/3 randomised trial. Lancet Oncol 14: 627-637.

Davies L, Mason JD, Roberts SA, Chan D, Reid TD, Robinson M, Gwynne S, Crosby TD, Lewis WG (2012) Prognostic significance of total disease length in esophageal cancer. Surg Endosc 26(10): 2810-2816.

Donovan JL, de Salis I, Toerien M, Paramasivan S, Hamdy FC, Blazeby JM (2014) The intellectual challenges and emotional consequences of equipoise contributed to the fragility of recruitment in six randomized controlled trials. J Clin Epidemiol; e-pub ahead of print 5 May 2014; doi:10.1016/j.jclinepi.2014.03.010.

Donovan J, Mills N, Smith M, Brindle L, Jacoby A, Peters T, Frankel S, Neal D, Hamdy F (2002) Improving design and conduct of randomised controlled trials by embedding them in qualitative research: ProtecT (prostate testing for cancer and treatment) study. BMJ 325: 766-770.

Donovan JL, Lane AJ, Peters TJ, Brindle L, Salter E, Gillatt D, Powell P, Bollina P, Neal DE, Hamdy FC. ProtecT Study Group (2009) Development of a complex intervention improved randomization and informed consent in a randomized controlled trial. J Clin Epidemiol 62: $29-36$.

Donovan JL, Paramasivan S, De Salis i, Toerien M (2014) Clear obstacles and hidden challenges: understanding recruiter perspectives in six pragmatic randomised controlled trials. Trials 15: 5 .

Glaser BG (1998) Doing Grounded Theory: Issues and Discussions. Sociology Press: Mill Valley, CA, USA.

Gwynne S, Hurt C, Evans M, Holden C, Vout L, Crosby T (2011) Definitive chemoradiation for oesophageal cancer-a standard of care in patients with non-metastatic oesophageal cancer. Clin Oncol 23: 182-188.

Hirst JSB, Gotley D, Thomas J, Barbour A (2011) Defining cure for esophageal cancer: analysis of actual 5-year survivors following esophagectomy. Ann Surg Oncol 18: 1766-1774.

Lagergren P, Fayers P, Conroy T, Stein HJ, Sezer O, Hardwick R, Hammerlid E, Bottomley A, Van Cutsem E, Blazeby JM. European Organisation for Research Treatment of Cancer Gastrointestinal and Quality of Life Groups (2007) Clinical and psychometric validation of a questionnaire module, the EORTC QLQ-OG25, to assess healthrelated quality of life in patients with cancer of the oesophagus, the oesophago-gastric junction and the stomach. Eur J Cancer 43: 2066-2073.

Lewin S, Glenton C, Oxman AD (2009) Use of qualitative methods alongside randomised controlled trials of complex healthcare interventions. BMJ 339: b3496.
McNair AG, Choh CT, Metcalfe C, Littlejohns D, Barham CP, Hollowood A, Falk SJ, Blazeby JM (2008) Maximising recruitment into randomised controlled trials: the role of multidisciplinary cancer teams. Eur J Cancer 44: 2623-2626.

National Oesophago-Gastric Cancer Audit (2012) The Third Annual Report, pp 1-69. NHS Information Centre: London, UK.

National Oesophago-Gastric Cancer Audit (2013) The Third Annual Report, pp 1-67. NHS Information Centre: London, UK.

Paramasivan S, Huddart R, Hall E, Lewis R, Birtle A, Donovan JL (2011) Key issues in recruitment to randomised controlled trials with very different interventions: a qualitative investigation of recruitment to the SPARE trial (CRUK/07/011). Trials 12: 78.

Parameswaran R, McNair A, Avery K, Berrisford RG, Wajed SA, Sprangers MA, Blazeby JM (2008) The role of health-related quality of life outcomes in clinical decision making in surgery for esophageal cancer: a systematic review. Ann Surg Oncol 15(9): 2372-2379.

Sobin LH, Wittekind C (2002) Oesophagus (ICD-O C15). In: TNM Classification of Malignant Tumours, Sobin LH, Wittkind C (eds) 6th edn, pp 60-69, Wiley: New York, NY, USA.

Stahl M, Stuschke M, Lehmann N, Meyer HJ, Walz MK, Seeber S, Klump B, Budach W, Teichmann R, Schmitt M, Schmitt G, Franke C, Wilke H (2005) Chemoradiation with and without surgery in patients with locally advanced squamous cell carcinoma of the esophagus. J Clin Oncol 23: 2310-2317.

Stahl M, Budach W, Meyer HJ, Cervantes A. On behalf of the ESMO Guidelines Working Group (2010) Esophageal cancer: Clinical Practice Guidelines for diagnosis, treatment and follow-up. Ann Oncol 21(Suppl 5): v46-v49.

Teoh A, Chiu P, Yeung W, Liu SY, Wong SK, Ng EK (2013) Long-term survival outcomes after definitive chemoradiation versus surgery in patients with resectable squamous carcinoma of the esophagus: results from a randomized controlled trial. Ann Oncol 24: 165-171.

Urba SG, Orringer MB, Turrisi A, Iannettoni M, Forastiere A, Strawderman M (2001) Randomized trial of preoperative chemoradiation versus surgery alone in patients with locoregional esophageal carcinoma. J Clin Oncol 19: 305-313.

van Hagen P, Hulshof MC, van Lanschot JJ, Steyerberg EW, van Berge Henegouwen MI, Wijnhoven BP, Richel DJ, Nieuwenhuijzen GA, Hospers GA, Bonenkamp JJ, Cuesta MA, Blaisse RJ, Busch OR, ten Kate FJ, Creemers GJ, Punt CJ, Plukker JT, Verheul HM, Spillenaar Bilgen EJ, van Dekken H, van der Sangen MJ, Rozema T, Biermann K, Beukema JC, Piet AH, van Rij CM, Reinders JG, Tilanus HW, van der Gaast A (2012) Preoperative chemoradiotherapy for esophageal or junctional cancer. N Engl J Med 366: 2074-2084.

This work is published under the standard license to publish agreement. After 12 months the work will become freely available and the license terms will switch to a Creative Commons AttributionNonCommercial-Share Alike 3.0 Unported License. 